\title{
UNA REVISIÓN SISTEMÁTICA DE LITERATURA SOBRE LA GESTIÓN DE LA PROPIEDAD INTELECTUAL EN INSTITUCIONES DE EDUCACIÓN SUPERIOR
}

\section{A systematic literature review of Intellectual Property Management in Higher Education Institutions.}

\author{
María Luisa Villalba Morales ${ }^{1}$, Francisco Javier Montoya ${ }^{2}$ \\ ${ }^{1}$ Ingeniera Industrial, M.Sc. en Ingeniería. Docente, Programa de Administración de Empresas, Universidad Católica de \\ Oriente.mvillalba@uco.edu.co. \\ ${ }^{2}$ Ingeniero Administrador, M.Sc. en Ingeniería. Docente Investigador Escuela de Administración, CEIPA Business School
} (Sabaneta-Antioquia, Colombia). francisco.montoya@ceipa.edu.co

(Recibido el 19 de Septiembre de 2018 y aprobado el 24 de Abril de 2019)

\begin{abstract}
Resumen
Ante los nuevos desafíos que afrontan las Instituciones de Educación Superior (IES) como generadoras de conocimiento, la gestión de su Propiedad Intelectual (PI) toma relevancia, con el fin de promover la obtención de resultados favorables de las creaciones que se gestan con sus recursos y que se esperan sean usadas por la industria y la sociedad. Por ello, ha sido de interés para algunos investigadores poder comprender la complejidad de estos sistemas de gestión. En este trabajo se expone el estado del arte de la gestión de la PI en el contexto colombiano, apoyado en casos de IES en el mundo y Latinoamérica. Entre los hallazgos principales se encuentran: 1) una evidente transición del reconocimiento de la importancia de la gestión, hacia la propuesta de modelos de gestión, y 2) la necesidad de profundizar en la definición de estructuras que soporten la complejidad y particularidad de una IES.
\end{abstract}

Palabras clave: Instituciones de Educación Superior - IES, Sistemas de Gestión, Propiedad Intelectual - PI, estado del arte

\begin{abstract}
Due to the new challenges faced by the Higher Education Institutions (HEIs) as generators of knowledge, the management of their Intellectual Property (IP) becomes relevant, in order to promote the obtaining of favorable results from the creations that are gestated with their resources and that are expected to be used by industry and society. Therefore, it has been interesting for some researchers to understand the complexity of these management systems. This paper exposes the state of the art of IP management in the Colombian context, supported by HEl cases in the world and Latin America. Among the main findings are: 1) a clear transition from the recognition of the importance of management to the proposal of management models, and 2) the need to deepen the definition of structures that support the complexity and particularity of an IES.
\end{abstract}

Keywords: Higher Education Institutions - HEls, Management Systems, Intellectual Property (IP),

\section{INTRODUCCIÓN}

Como una de las consecuencias del avance tecnológico y la importancia del conocimiento como activo, la propiedad intelectual (PI) se ha convertido en un factor clave de competitividad para las empresas [1], lo que ha desatado la necesidad de realizar una adecuada gestión de aquellos recursos basados en el conocimiento, de tal modo, que los esfuerzos e inversiones enfocadas en la protección y explotación de la propiedad intelectual se acojan a buenas prácticas de administración.

Aunque la anterior afirmación haga referencia a las empresas, lo cierto es que esta realidad no excluye a las instituciones de educación superior (IES), las cuales también se encuentran inmersas en los sistemas económicos y de innovación, por ser generadoras de conocimiento a través de sus acciones misionales: la 
docencia, la investigación y la extensión; a las cuales, en las últimas décadas se le han sumado el emprendimiento y la creación de spin offs [2]. Por ello, y entre otras razones operacionales, se ven en la necesidad de contar con un sistema apropiado para la protección de su propiedad intelectual [3].

En este sentido, un sin número de IES en el mundo han ido tomando conciencia y han podido realizar acciones que conlleven a la adecuada estructuración y adaptación de un sistema de gestión de la propiedad intelectual, partiendo desde políticas claras y objetivos alcanzables, hasta llegar a la implementación de procesos e instrumentos de gestión adecuados a las características de cada universidad.

Específicamente, en el caso de las IES colombianas, se han realizado diferentes trabajos para medir el nivel de gestión de la PI. Entre estos trabajos destacan los realizados por [3], [4] y [5], que de forma aglomerada (sin definir universidades específicas para el estudio), aportan a la comprensión del panorama colombiano de la gestión la PI en IES.

No obstante, también se evidencia un alto número de estudios de casos específicos, es decir, trabajos en los cuales el objeto de estudio corresponde a un número finito de IES. Son estos casos los de interés de los autores, quienes han plasmado en este trabajo un compendio de dichos casos, con el fin de evidenciar las tendencias en cuanto a la Gestión de la PI, las metodologías empleadas y otros aspectos que sean relevantes en torno a la temática.

\section{METOdOLOGÍA}

La metodología empleada en este trabajo es de carácter cualitativa sustentada en una investigación observacional y retrospectiva, con el objetivo de sintetizar investigaciones realizadas en el ámbito de la gestión de la propiedad intelectual en IES, teniendo en cuenta el contexto mundial, latinoamericano y colombiano. Para ello se siguieron las siguientes etapas:

\subsection{Definición de la pregunta a responder}

La pregunta planteada para ser resuelta con esta investigación se centra en la necesidad de conocer el rumbo que actualmente están tomando las investigaciones desarrolladas en cuanto a la gestión de la propiedad in- telectual en IES, con el fin de identificar las tendencias en cuanto a enfoques y metodologías para valorar y/o analizar los diferentes casos estudiados y con ello poder identificar aspectos claves para las instituciones que aún no han iniciado el proceso de gestión de la propiedad intelectual. Con base en ello, la pregunta que se busca responder en esta investigación es:

¿Es posible identificar una evolución en los casos de estudio sobre gestión de la PI en IES colombianas en lo referente a objeto de estudio y enfoque?

\subsection{Definición de las características de los casos estudio}

Se incluyeron dentro de esta investigación los trabajos que cumplieran con los siguientes criterios:

- Tipo de diseño: se incluyen trabajos de análisis cualitativo y/o cuantitativo

- Tamaño de muestra: se incluyen casos que sean específicos (una sola IES), como también casos que muestren tendencias aglomeradas.

- Años de publicación: a partir del año 2008, para completar un periodo de análisis de ocho (8) años.

\subsection{Búsqueda y selección de los casos}

Se definieron tres fuentes de búsqueda para la identificación de los casos: 1) publicaciones en eventos académicos, 2) publicaciones de revistas científicas y 3) publicaciones de informes de entidades de fomento de la propiedad intelectual. La búsqueda se realizó en un periodo de tres meses: noviembre 2016 a enero de 2017. En total se seleccionaron 14 casos y cinco (5) trabajos de enfoque general, distribuidos así:

- Casos en el mundo: tres (3) casos.

- Casos en Latinoamérica: dos (2) casos

- Casos en Colombia: nueve (9) casos, más cinco (5) trabajos de enfoque general

\subsection{Análisis de los hallazgos}

Los hallazgos fueron analizados de dos formas:

- Casos en el mundo y Latinoamérica. Estos casos se revisaron y compararon con los documentos 
disponibles, para algunos de ellos se encontró un solo documento.

- Casos colombianos. Se definieron cinco criterios de análisis (tipo de documento, año de publicación, número de IES estudiadas, metodología empleada y aspectos relevantes de la gestión de la PI)

\section{RESULTADOS}

De acuerdo a los casos hallados y analizados, se muestran los resultados, según el alcance de los mismos.

\subsection{En el mundo}

A continuación se describen tres (3) casos sobre gestión de la propiedad intelectual en IES de tres países.

\subsubsection{Tailandia}

Para las IES en Tailandia, los autores del trabajo [6] realizaron una propuesta general de estructuración de un departamento de propiedad intelectual y una serie de capacitaciones a los investigadores sobre la temática, en el año 2001.

La problemática que sustenta esta propuesta era que la mayoría de las universidades del país tienen dentro de sus campos de acción la biotecnología, área en la cual, las universidades generan valiosos conocimientos académicos; pero los estaban perdiendo en las negociaciones con las empresas extranjeras con las que realizaban la investigación [6].

En este sentido, la propuesta de los autores consistió en la implementación de cursos de gestión de la propiedad intelectual y el establecimiento de un departamento de propiedad intelectual, con el fin de estimular y activar a profesores e investigadores a tomar medidas para proteger sus descubrimientos en el área de la biotecnología [6].

Con base en ello, se identifica que los esfuerzos estaban orientados a la sensibilización de los investigadores y realizar los primeros esfuerzos por contar con una estructura visible de gestión. Esta condición se presentó en el año 2001, pero se asemeja al estado identificado en algunas de las IES colombianas entre el 2008 y el 2012, de acurdo a los resultados de los estudios realizados por [4], [5], [7] y [8].

\subsubsection{Estados Unidos}

Las Universidades en Estados Unidos, en su mayoría cuentan con políticas de propiedad intelectual. Un estudio realizado en el 2008 , en el que se encuestaron científicos de nueve (9) universidades, concluye que las políticas universitarias, en cuanto a Propiedad Intelectual, son vehículos fundamentales para la gestión de las relaciones entre la industria y las universidades, con el fin de mejorar el largo plazo el bienestar económico y social de las universidades, sus científicos y empresas del sector privado. Lo anterior, teniendo en cuenta que: 1) En Estados Unidos existe una larga historia de registro y uso de patentes por parte de las Universidades (Ley Bayh-Dole). 2) La existencia de políticas de propiedad intelectual deben facilitar la transferencia tecnológica, permitir ingresos a la universidad, sin que se pierda la libertad académica y la promoción para la continuidad en la generación y perfeccionamiento de conocimiento [2], [9].

Pero no solo se quedan con políticas, también se han definido mecanismos de compensación para los profesores-investigadores, a través de las regalías que se obtienen de la comercialización de los productos desarrollados. Ejemplo de estas universidades son las Universidades de "California, Stanford, Columbia, Wisconsin, Washington, Michigan, lowa, Massachussets, Florida, Harvard, Cornell y Colorado" [10].

Con base en lo anterior, se resalta el avance que tienen las universidades en Estados Unidos en cuanto a la Gestión de la Propiedad Intelectual. Lo cual se debe, no sólo a que las universidades identifican la importancia de gestionar la propiedad intelectual, sino también a los lineamientos definidos en las leyes de protección.

\subsubsection{Indonesia}

De este país se tiene el caso de Bogor Agricultural University, una de las principales universidades, en un contexto de economía emergente, que juega un papel importante en el campo de la agricultura del país y tuvo un cambio estructural que le permitió llegar a ser una universidad basada en la investigación de clase mundial, lo que implica gestionar la innovación y la investigación. Por lo cual, la gestión de la propiedad intelectual toma relevancia, y para la Universidad fue necesario establecer mecanismos institucionales para gestionar los resultados de la investigación (investigaciones compartida, licencias, o emprendimientos tecnológicos)

Rev. Ing. Investig. Desarro. Vol.18 (2). pp. 47-59, Julio-Diciembre. 2018, Sogamoso-Boyacá, Colombia. 
y cuenta con una oficina de derechos de propiedad intelectual y publicaciones, la cual tuvo los siguientes desafíos: 1) Escaso personal con conocimientos en propiedad intelectual, habilidades en transferencia y marketing; 2) Poco conocimiento de los investigadores en protección y comercialización de los resultados de investigaciones, 3) Falta de una política de propiedad intelectual, y resultados en los laboratorios sin generación de prototipos [2].

Con base en ello se definió una política de propiedad intelectual en el año 2004, la cual incluye los actores que pueden participar en la generación de propiedad, la protección, la comercialización y los beneficios recibidos. Las acciones en este ámbito han evolucionado desde entonces y actualmente las políticas abarcan los trabajos en redes por fuera de la universidad [2].

Este caso puede convertirse en modelo de gestión replicable para las universidades colombianas, puesto que comparten el contexto de economías emergentes, la fortaleza en el sector de la agricultura y la transformación de las universidades hacia el enfoque investigativo y de emprendimiento. Adicional a ello, el caso refleja el trabajo que se ha venido realizando en la Universidad desde el año 2000 para fortalecer su sistema de Gestión de la propiedad intelectual y que éste se adapte a las condiciones cambiantes del entorno.

\subsection{En Latinoamérica}

A continuación se describen cuatro (4) casos sobre gestión de la propiedad intelectual en instituciones de educación superior de diferentes países latinoamericanos.

\subsubsection{Red de Propiedad Intelectual e Industrial en Latinoamérica (Red PILA)}

La red PILA tiene como misión "Fortalecer la cooperación entre las IES de América Latina en todos los aspectos relacionados a Propiedad Intelectual (PI) e innovación, promoviendo la ayuda mutua para la consolidación de acciones de gestión de Propiedad Intelectual [11] Entre el 2009 y 2011 realizó un proyecto para diagnosticar el estado de la gestión de la propiedad intelectual en las Universidades, a través de la metodología AIDA, la cual se basa en el supuesto que existen cuatro (4) niveles de madures para la gestión de la propiedad intelectual: Atención, Interés, Deseo y Acción. En este proyecto participaron 17 países (Argentina, Bolivia, Brasil, Chile,
Colombia, Costa Rica, Cuba, Ecuador, El Salvador, Honduras, México, Nicaragua, Panamá, Paraguay, Perú, Uruguay y Venezuela) [12].

En la investigación participaron 147 universidades, lo que permitió obtener valores en la escala de 1 a 10, siendo 10 el valor más alto que se puede obtener en las categorías de conocimiento, protección, gestión y explotación. Estos valores fueron ponderados y se obtuvo la media para cada país, donde el valor más alto, lo alcanzó Brasil $(6,51)$ y el más bajo lo obtuvo Paraguay $(0,78)$. En la Tabla 1 se muestran los valores para cada país.

Las principales conclusiones de este estudio se centran en la falta de formación a profesores, administrativos y estudiantes en temas de propiedad intelectual, bajos niveles de explotación de la propiedad intelectual y carencia de estructuras formales para la gestión de la propiedad intelectual en la mayoría de las universidades. Lo anterior, sustentado en factores nacionales, institucionales y operativos [7].

De este proyecto, también se realizaron estudios específicos por países. El caso colombiano ha sido tomado dentro de los documentos de análisis de este trabajo.

\subsubsection{Brasil}

El inicio de la protección de los resultados de las investigaciones en las universidades públicas en Brasil inició en la década de los 80, y para el año 2007 el interés por la protección de la propiedad intelectual estaba en aumento, evidenciándose en el número de creaciones protegidas (Sabino \& Ayuso, 2012).

Los autores [13] en el 2012 realizan un análisis comparativo entre la Universidad Federal de Goias y la Universidad de Brasilia, con el fin de identificar si las universidades presentaban diferencias en cuanto a la gestión de la propiedad intelectual. Aunque emiten conclusiones sobre la existencia de diferencias significativas entre las universidades y la necesidad de considerar aspectos claves como la normalización interna, el apoyo de la dirección y el cambio de cultura, las variables empleadas para el estudio se limitaron a la existencia de políticas en propiedad intelectual, el perfil de los investigadores y el número de patentes registradas. 
Tabla 1. Puntuación por país en el diagnóstico de gestión de la Propiedad Intelectual del proyecto PILA

\begin{tabular}{|c|c|c|c|c|c|}
\hline \multirow{2}{*}{ Países } & \multicolumn{5}{|c|}{ NIVEL AIDA } \\
\cline { 2 - 6 } & Conocimiento & Protección & Gestión & Explotación & Media \\
\hline Argentina & 8,33 & 4,19 & 5,15 & 3,54 & 5,3 \\
\hline Bolivia & 2,44 & 0,96 & 1,26 & 1,15 & 1,45 \\
\hline Brasil & 8,81 & 6,19 & 6,58 & 4,45 & 6,51 \\
\hline Chile & 6,06 & 4,45 & 4,31 & 3,47 & 4,57 \\
\hline Colombia & 8,06 & 3,89 & 3,68 & 2,91 & 4,63 \\
\hline Costa Rica & 7,71 & 4,59 & 4,34 & 3,65 & 5,08 \\
\hline Cuba & 7,01 & 4,48 & 3,56 & 2,99 & 4,51 \\
\hline Ecuador & 7 & 4,43 & 4,52 & 3,26 & 4,8 \\
\hline El Salvador & 5,08 & 1,62 & 1,4 & 1,58 & 2,42 \\
\hline Honduras & 5,02 & 1,54 & 0,77 & 1,14 & 2,12 \\
\hline México & 6,18 & 4,84 & 4,94 & 3,9 & 4,96 \\
\hline Nicaragua & 5,5 & 3,01 & 3,15 & 2,53 & 3,55 \\
\hline Panamá & 7,68 & 4,05 & 3,98 & 3,96 & 4,91 \\
\hline Paraguay & 1,87 & 0,45 & 0,46 & 0,33 & 0,78 \\
\hline Perú & 6,58 & 4,16 & 3,05 & 2,94 & 4,18 \\
\hline Uruguay & 7,18 & 3,6 & 2,78 & 1,88 & 3,86 \\
\hline Venezuela & 6,79 & 3,01 & 2,82 & 2,22 & 3,71 \\
\hline Promedio & $\mathbf{6 , 3 1}$ & $\mathbf{3 , 5}$ & $\mathbf{3 , 3 4}$ & 2,76 & 3,96 \\
\hline & & & & & \\
\hline
\end{tabular}

Tomado de [7] pág. 23.

\subsection{Colombia}

El estudio de la gestión de la propiedad intelectual en las IES en Colombia tiene dos directrices. Se pueden encontrar trabajos enfocados al análisis general a nivel país, sea con el fin de mostrar el estado general de la gestión, o analizar factores que influyen en ella. Como también, se encuentran trabajos que buscan evidenciar casos específicos, de una o varias IES en cuanto a su situación particular para la gestión de la Propiedad Intelectual.
En la Tabla 2 se muestra el paralelo de los estudios analizados; de los cuales, seis (6) son estudios a nivel nacional, mientras que 10 son casos, ya sean aglomerados o específicos.

Los estudios de análisis general, han sido publicados entre el año 2011 y el año 2015, mientras que los casos se encuentran entre el año 2006 y 2016, con comportamiento variable en cuanto a la frecuencia anual (ver Gráfica 1). 
Una revisión sistemática de literatura sobre la gestión de la propiedad intelectual en Instituciones de Educación Superior

Tabla 2. Estudios de la Gestión de la Propiedad Intelectual en Colombia

\begin{tabular}{ll}
\hline & \multicolumn{1}{c}{ Análisis general } \\
\hline$[10]$ & $\begin{array}{l}\text { El estado de La Propiedad Intelectual } \\
\text { en las Universidades Colombianas. }\end{array}$ \\
& \\
& \\
& Modelos y mecanismos de \\
& interacción universidad-empresa- \\
& Estado: retos para las universidades \\
& colombianas \\
& La Propiedad Intelectual en las \\
& Universidades: Nuevos centros \\
de innovación, emprendimiento \\
y transferencia de tecnología; \\
titularidad de derechos y legislación.
\end{tabular}

[16] Guía Estretégica de Propiedad Intelectual. Universidad Empresa

[3] La Gestión Universitaria de la Propiedad Intelectual en Colombia

\begin{tabular}{ll}
\hline \multicolumn{3}{c}{ Casos } \\
\hline$[18]$ & $\begin{array}{l}\text { Algunos efectos de la Gestión } \\
\text { Tecnológica en la producción } \\
\text { Industrial y el papel de la educación } \\
\text { para su apropiación en Colombia }\end{array}$
\end{tabular}

[4] La propiedad intelectual en las economías universitarias

[8] La Gestión de la Propiedad Intelectual en las Universidades Colombianas: El caso de la Universidad Industrial de Santander

[5] El Patentamiento Universitario en Colombia

Determinantes de la transferencia de propiedad industrial al sector productivo en universidades públicas colombianas

[23] Diagnóstico del Sistema de Gestión de la Propiedad Intelectual Universitaria: Caso Universidad de San Buenaventura

[24] Importancia de un Modelo de Gestión de Propiedad Intelectual en una Institución De Educación Superior: Caso Instituto Tecnológico Metropolitano

Fuente: Elaboración propia 
Gráfica 1. Frecuencia anual de trabajos enfocados en la Gestión de la Propiedad Intelectual.

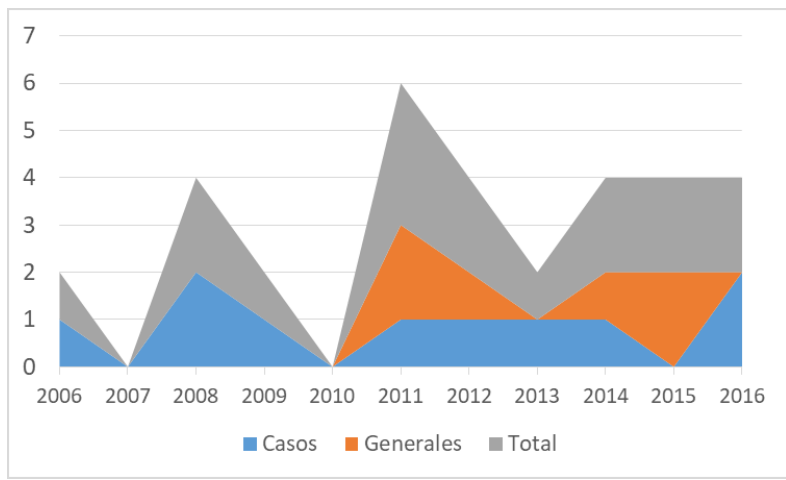

Fuente: Elaboración propia

\subsubsection{Trabajos de análisis general}

Los trabajos de análisis general a nivel país buscan aportar al conocimiento de la Gestión de la Propiedad Intelectual en las IES colombianas, sin entrar en detalles de las mismas. Este tipo de trabajo tuvo su mayor producción en el año 2011 (aunque entre los años 2008 y 2010 ya se contaba con estudios de casos específicos).

El trabajo [21] ofrece un panorama del nivel de conocimiento, protección, gestión y explotación de la Propiedad Intelectual. Entre los principales hallazgos se resalta la carencia generalizada de políticas y un alto desconocimiento de la temática por parte de los integrantes de las IES. Estos resultados desatan el interés por la realización de estudios a profundidad y comparativos entre IES, con el fin de generar planes y cursos de capacitación. Con base a ello, se esperaría un crecimiento de los estudios en Gestión de la Propiedad Intelectual, sin embargo, para los años próximos, el número de trabajos generales disminuyó. En el 2011 también se realizó el estudio de [14] quienes, a través de un análisis de literatura identificaron los retos a los que se ven enfrentadas las IES colombianas, para interactuar con la empresa y el estado. Estos retos impactan directamente en la gestión de la propiedad intelectual, principalmente por la necesidad de políticas de protección, las cuales deben mejorar con relación a las establecidas en las IES de países desarrollados (ejemplo: Estados Unidos).

Con base en ello, toma relevancia el trabajo realizado por [15], puesto que busca comprender las implicaciones legales que conllevan las nuevas creaciones y dinámicas de generación y transferencia de conocimiento (Centros de innovación, emprendimiento y transferencia tecnológica). Este trabajo, a diferencia de los anteriores, se centra en la aplicación de la normatividad que rige la propiedad intelectual para Colombia, más que para definir el estado de gestión de las IES.

Realizando un salto de dos años, se lanza una Guía de actuación [16] en temas Propiedad Intelectual, para ofrecer a universidades y empresas, pautas requeridas para la transferencia tecnológica y el trabajo en conjunto entre ellas. Aunque esta guía no indica el estado de las IES en cuanto a su nivel de gestión, contextualiza y describe las variables que se deben contemplar para garantizar la protección de la propiedad intelectual de las IES.

Para el año 2015, se realizaron dos publicaciones importantes. Una corresponde al trabajo realizado por [3] y la otra al trabajo realizado por [17] De estos dos (2) trabajos, el primero está orientado a la descripción del contexto en el cual se ven inmersas las IES y que afectan la propiedad intelectual (Sistemas de gestión de PI, el Sistema Nacional de Innovación de Colombia, histórico de protección y uso de la PI generara en las Universidades, y buenas prácticas en gestión por parte de las IES); mientras que el segundo pone en manifiesto las falencias que tiene el país en cuanto la asignación de recursos y mecanismos que permitan mejorar la interacción entre los actores del Sistema Nacional de Innovación, con base en las políticas gubernamentales relacionadas con la propiedad intelectual.

\subsubsection{Casos}

\section{Generalidades}

El aporte al conocimiento en cuanto a Gestión de la PI en las IES en Colombia, es realizado a través de diferentes tipos de documentos tal como se muestra en la Gráfica 2. De acuerdo a la distribución obtenida, se puede identificar la importancia de la temática en Colombia, puesto que además de contar con publicaciones en revistas indexadas, también se cuenta con informes de proyectos y ponencias. A esto se suma, que uno de los casos fue presentado en Congreso y por su impacto fue publicado (dos años después) en la revista Journal of Technology Management \& Innovation, como un Special Issue ALTEC [5]. Sin embargo, la mayoría de los estudios son de nivel exploratorio (incluyendo los más actuales), lo que evidencia que es un tema en crecimiento para

Rev. Ing. Investig. Desarro. Vol.18 (2). pp. 47-59, Julio-Diciembre. 2018, Sogamoso-Boyacá, Colombia. 
el contexto colombiano y estos trabajos apuntan a la generación de evidencia empírica. La cual tiene como fuente directa las IES, principalmente de casos puntuales o máximo tres (3) instituciones. En la Gráfica 3 se muestra la población de IES estudiadas.

Gráfica 2. Tipos de documentos de los casos publicados

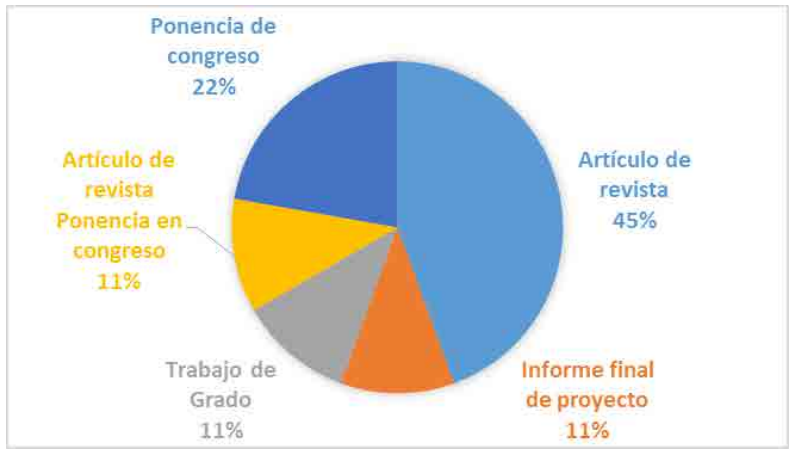

Fuente: Elaboración propia

Gráfica 3. Número de IES estudiadas en los casos.

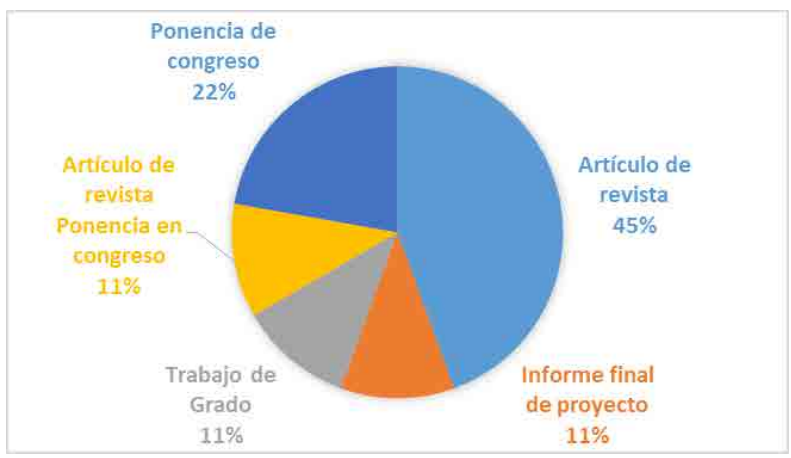

Fuente: Elaboración propia

De acuerdo al Sistema Nacional de Información de la Educación Superior, para el año 2017 existen en Colombia 289 IES activas. De los casos estudiados se pudieron identificar 17 IES, lo cual representa el 5.9\% de la población total.

\section{Metodologías empleadas}

Los diseños metodológicos empleados en los nueve

(9) casos mezclan el enfoque cualitativo y cuantitativo.

Lo que permite emitir valoraciones comparables entre las instituciones que fueron analizadas de forma aglomerada. Las fuentes definidas para las investigaciones principalmente corresponden a la información documental y el personal directivo e investigativo de las IES. Excepto, para aquellos casos en los que las universidades no son definidas, sino que se hace estudios de bases de datos de patentes o de marca.

En cuanto a los métodos de recolección y análisis de la información, se destaca la metodología AIDA y la metodología propuesta por [10]. La primera es de enfoque cuantitativo, usada en tres casos: [8], [20] y [21]. La segunda es de enfoque cualitativo, usada en un caso colombiano [24] y por los autores proponentes [1] en un caso mexicano. Ambas se describen en la Tabla 3. El resto de los casos se basan en metodologías sustentadas en el método científico o análisis de casos, en los cuales se incluye la realización de entrevistas o la revisión del histórico de patentes nacionales.

\section{Aspectos relevantes de la Gestión de la PI}

De acuerdo a la información entregada por los casos, se definieron dos (2) criterios de clasificación, que permiten identificar los aspectos relevantes en los cuales están siendo analizadas las IES Colombianas. Estos son: el reconocimiento de la importancia de la gestión de la PI por parte de las directivas de las IES; y la definición de políticas o modelos para la gestión. A continuación se describen los hallazgos de cada aspecto relevante.

- Reconocimiento de la importancia de la Gestión.

En cuanto a este criterio, se identificó que en la totalidad de los casos, algunos con mayor profundidad que otros, abordan o indagan sobre la importancia de realizar gestión de la PI en las IES y si las directivas reconocen dicha importancia. El nivel de profundidad se define de acuerdo al enfoque de cada trabajo, puesto que tres de ellos (los realizados entre el 2008 y el 2009) están orientados principalmente a exteriorizar y comprender el nivel de concientización e importancia que tienen las IES estudiadas sobre la Gestión de la PI; mientras que el resto de casos tienen otros enfoques, donde este tópico es importante, pero no el centro de la investigación. En la Tabla 4 se describen los enfoques de los trabajos. 
Tabla 3. Métodos para evaluación de la gestión de la PI en IES

\begin{tabular}{|c|c|c|}
\hline Metodología & Enfoque & Descripción \\
\hline \multirow{13}{*}{$\begin{array}{l}\text { La metodología } \\
\text { AIDA } \\
{[20]}\end{array}$} & \multirow{13}{*}{ Cuantitativo } & $\begin{array}{l}\text { El método se basa en la suposición de que, esquemáticamente, la } \\
\text { explotación óptima de la PI se desarrolla en los siguientes pasos sucesivos } \\
\text { (niveles de AIDA): }\end{array}$ \\
\hline & & A \\
\hline & & Atención \\
\hline & & Conocimiento / conciencia \\
\hline & & $\mathbf{I}$ \\
\hline & & Interés \\
\hline & & Protección \\
\hline & & D \\
\hline & & Deseo \\
\hline & & Gestión \\
\hline & & A \\
\hline & & Acción \\
\hline & & Explotación \\
\hline \multirow{10}{*}[10]{} & \multirow{10}{*}{ Cualitativo } & $\begin{array}{l}\text { La gestión de la propiedad intelectual en universidades y centros de } \\
\text { investigación, consta de nueve (9) componentes. }\end{array}$ \\
\hline & & 1. Promoción de la inventiva \\
\hline & & 6. Valuación de PI \\
\hline & & 2. Selección y admón. de proyectos \\
\hline & & 7. Licenciamiento de la tecnología propia \\
\hline & & 3.Inteligencia tecnológica competitiva \\
\hline & & 8. Auditoría de propiedad intelectual \\
\hline & & 4. Adquisición licencias para investigación \\
\hline & & 9.Vigilancia del patrimonio tecnológico \\
\hline & & 5. Estrategia de protección \\
\hline
\end{tabular}

Fuente: Elaboración propia 
Una revisión sistemática de literatura sobre la gestión de la propiedad intelectual en Instituciones de Educación Superior

Tabla 4. Enfoques de los casos estudiados.

\begin{tabular}{|c|c|c|}
\hline Año & Autores & Enfoque \\
\hline 2006 & Alvarado & $\begin{array}{l}\text { Efectos de la educación técnica, universitaria y tecnológica en la gestión tecnológica } \\
\text { empresarial (transferencia y aplicación de conocimiento) }\end{array}$ \\
\hline 2008 & $\begin{array}{l}\text { Vallejo \& } \\
\text { Álvarez }\end{array}$ & $\begin{array}{l}\text { Importancia de la propiedad intelectual desde la importancia del enfoque en la investigación } \\
\text { de las Universidades y la transferencia de resultados de investigaciones al sector industrial. }\end{array}$ \\
\hline 2008 & García & Conciencia de la importancia de la propiedad intelectual \\
\hline 2009 & $\begin{array}{c}\text { Red de } \\
\text { Propiedad } \\
\text { Intelectual en } \\
\text { Latinoamérica }\end{array}$ & Nivel de concientización en los instrumentos de PI \\
\hline 2011 & $\begin{array}{l}\text { González \& } \\
\text { Jaime }\end{array}$ & $\begin{array}{l}\text { Panorama general sobre el estado de la Propiedad Intelectual en las Universidades } \\
\text { Colombianas }\end{array}$ \\
\hline \multirow[t]{2}{*}{2012} & González, D. & $\begin{array}{l}\text { Identificación de la Gestión de la propiedad intelectual para facilitar la transferencia } \\
\text { tecnológica, a través de las etapas: Identificar, procesar - proteger, usar - transferir, capacitar. }\end{array}$ \\
\hline & & Propuesta de un modelo de gestión \\
\hline $\begin{array}{l}2011 \\
2013\end{array}$ & $\begin{array}{l}\text { González \& } \\
\text { Jaime }\end{array}$ & Nivel de patentamiento de las universidades Colombianas \\
\hline 2014 & $\begin{array}{c}\text { Morales, } \\
\text { Sanabria, \& } \\
\text { Plata }\end{array}$ & $\begin{array}{l}\text { Factores que han incidido en la transferencia de propiedad industrial universitaria al sector } \\
\text { productivo }\end{array}$ \\
\hline 2016 & $\begin{array}{l}\text { Montoya \& } \\
\text { Villalba }\end{array}$ & Existencia de políticas y mecanismos de protección de la PI \\
\hline 2016 & $\begin{array}{l}\text { Gaviria \& } \\
\text { Londoño }\end{array}$ & Aplicación de un modelo de Gestión de PI existente al caso del ITM \\
\hline
\end{tabular}

Fuente: Elaboración propia

- Políticas, modelos y estructura organizacional para la gestión

Este aspecto relevante, representa para las IES un escalón superior al reconocimiento de la importancia de la gestión de la $\mathrm{PI}$, puesto que contar con políticas, modelos de gestión y la estructura organizacional para hacerlo, es una evidencia de que las IES han desarrollado la conciencia sobre la importancia de gestionar su PI. En este sentido, desde el año 2009, los casos estudiados, incluyen aspectos como la existencia de políticas de PI y su conocimiento por parte del personal de la IES, la aplicación o propuesta de modelos para la gestión de PI y por último, la identificación, propuesta o evaluación de mecanismos o instrumentos para realizar la gestión. Excepto aquel trabajo que se enfoca en el patentamiento, tal como se muestra en la Tabla 5.

De los trabajos analizados, el de mayor profundidad corresponde al realizado por [8], puesto que además de evidenciar el estado de la IES, propone un modelo de gestión, el cual se alinea con las recomendaciones propuestas por [6] para las universidades en Tailandia. Sin embargo, se evidencia un mayor nivel de gestión para la universidad colombiana, que puede ser resultado de la evolución temática de los 11 años de las publicaciones y el estado en el cual se encontraba la Universidad de acuerdo a la infraestructura con la que contaba para promover la $\mathrm{PI}$.

Por otro lado, se tiene que cada trabajo se centra en aspectos específicos para la definición de la estructura para promover la $\mathrm{PI}$, de los cuales no se puede identificar una correspondencia total entre los trabajos, pero si aspectos importantes, tales como la existencia de políticas marco en $\mathrm{PI}$, la acciones para promover la inventiva, los procesos para la protección y la transferencia tecnológica. Esto se debe al enfoque de cada trabajo y los referentes teóricos que se consideraron para el desarrollo de cada estudio. 


\section{CONCLUSIONES.}

El estudio de la gestión de la PI es una herramienta para el entendimiento del papel de la PI el marco de acción de las IES como generadoras de conocimiento, su participación activa en los sistemas de innovación de los países y su inminente transformación en ambos aspectos. Esto se refleja en los enfoques de los estudios que tratan esta temática.

Como se evidencia para el caso colombiano, los estudios de hace más de ocho (8) años se enfocaban en identificar la importancia que tiene la gestión de la $\mathrm{PI}, \mathrm{y}$ cómo las IES van tomando conciencia de ello. Este aspecto aún es relevante a la fecha, pero ya no es el eje central de las investigaciones, las cuales se centran en la identificación de modelos de gestión o mecanismos, que puedan garantizar mejores resultados en cuanto a protección y explotación de la PI, y al mismo tiempo evidenciar el estado o el nivel de la gestión de la PI, el cual se encuentra por debajo de las IES de otros países como Estados Unidos, pero sin diferencias significativas o superior a las IES en algunos países de Latinoamérica.

Es por ello, que la realización de estudios en torno a la temática sigue siendo pertinente, siempre y cuando las investigaciones se orienten a profundizar en el que hacer de las IES y permitan generar propuestas de mejora (modelos o instrumentos para la gestión), que se adapten a las condiciones de las IES colombianas. Lo anterior implica nuevas propuestas metodológicas y la validación a través de casos aplicados y que se consideren los hallazgos previos y las directrices globales (como las emitidas por la OMPI) y las nacionales (las emitidas por la SIC).

Tabla 5. Trabajos enfocados en Políticas, modelos de gestión e infraestructura para la gestión de la PI

\begin{tabular}{|c|c|c|c|c|}
\hline Año & Autores & $\begin{array}{l}\text { Modelos de } \\
\text { Gestión }\end{array}$ & Políticas & Estructura para promover la PI \\
\hline 2008 & Vallejo \& Álvarez & & & \\
\hline 2008 & García & & & \\
\hline 2009 & $\begin{array}{l}\text { Red de Propiedad } \\
\text { Intelectual en } \\
\text { Latinoamérica }\end{array}$ & & $\begin{array}{l}\text { Miden el nivel } \\
\text { conocimiento }\end{array}$ & $\begin{array}{l}\text { Miden el nivel Gestión (criterios de la metodología } \\
\text { AIDA) }\end{array}$ \\
\hline 2011 & González \& Jaime & & $\begin{array}{l}\text { Miden el nivel } \\
\text { conocimiento }\end{array}$ & $\begin{array}{l}\text { Miden el nivel Gestión (criterios de la metodología } \\
\text { AIDA) }\end{array}$ \\
\hline 2012 & González, D. & Propone & $\begin{array}{l}\text { Se enfocan en } \\
\text { la existencia } \\
\text { de Políticas de } \\
\text { investigación }\end{array}$ & $\begin{array}{l}\text { Se identifican aspectos como: } \\
\text { - Capacitación } \\
\text { - Monitoreo de Creaciones } \\
\text { - Incentivos para los investigadores } \\
\text { - Tramites de protección } \\
\text { - Creación de la Vicerrectoría de Investigación y } \\
\text { - Extensión } \\
\text { - Programa de apoyo a solicitudes de registro de PI }\end{array}$ \\
\hline $\begin{array}{l}2011 \\
2013\end{array}$ & González \& Jaime & & & \\
\hline 2014 & $\begin{array}{c}\text { Morales, } \\
\text { Sanabria, \& Plata }\end{array}$ & & $\begin{array}{l}\text { Se nombra la } \\
\text { existencia de } \\
\text { reglamentos } \\
\text { de PI }\end{array}$ & $\begin{array}{l}\text { Se nombran las capacidades de las Universidades: } \\
\text { - Motivación para patentar } \\
\text { - Aprendizaje de procesos en torno a la } \\
\text { - transferencia } \\
\text { - Relación activa con el entorno } \\
\text { - Base financiera } \\
\text { - Unidades de Transferencia }\end{array}$ \\
\hline
\end{tabular}

Rev. Ing. Investig. Desarro. Vol.18 (2). pp. 47-59, Julio-Diciembre. 2018, Sogamoso-Boyacá, Colombia. 


\begin{tabular}{|c|c|c|c|c|}
\hline Año & Autores & $\begin{array}{l}\text { Modelos de } \\
\text { Gestión }\end{array}$ & Políticas & Estructura para promover la PI \\
\hline 2016 & $\begin{array}{c}\text { Montoya \& } \\
\text { Villalba }\end{array}$ & & $\begin{array}{c}\text { Se mide la } \\
\text { Existencia } \\
\text { y nivel de } \\
\text { conocimiento }\end{array}$ & $\begin{array}{l}\text { Se identifican aspectos como: } \\
\text { - Instrumentos de gestión: Vigilancia, } \\
\text { confidencialidad, contratos, entre otros }\end{array}$ \\
\hline 2016 & $\begin{array}{l}\text { Gaviria \& } \\
\text { Londoño }\end{array}$ & $\begin{array}{c}\text { Evalúa } \\
\text { modelos y } \\
\text { aplica uno } \\
\text { específico al } \\
\text { Caso (Aplican } \\
\text { el modelo } \\
\text { de Luna } \\
\& \text { Solleiro } \\
\text { (2007) }\end{array}$ & $\begin{array}{c}\text { Evalúa la } \\
\text { Existencia } \\
\text { y nivel de } \\
\text { conocimiento }\end{array}$ & $\begin{array}{l}\text { Se identifican aspectos como: } \\
\text { - Promoción a la inventiva } \\
\text { - Selección y Administración de Proyecto } \\
\text { - Inteligencia Tecnológica Competitiva } \\
\text { - Adquisición de Licencias para la Investigación } \\
\text { - Estrategias de Protección } \\
\text { - Valoración de la Propiedad Intelectual } \\
\text { - Licenciamiento de la Tecnología Propia } \\
\text { - Auditoría de Propiedad Intelectual } \\
\text { - Vigilancia del Patrimonio Tecnológico }\end{array}$ \\
\hline
\end{tabular}

Nota: los cuadros resaltados con gris, representa que no contaban con la información requerida

Fuente: Elaboración propia

\section{REFERENCIAS}

[1] C. Atehortúa y E. Yepes, «Gestión Estratégica de Propiedad Intelectual para la inserción en redes de la economia del conocimiento, " de Guía estratégica de Propiedad Intelectual Universidad Empresa, Medellín, Vallejo Editores, 2014, pp. 29-47.

[2] J. Payumo, P. Arasu, A. Fauzi, I. Siregar y D. Noviana, "An entrepreneurial, research-based university model focused on intellectual property management for economic development in emerging economies: The case of Bogor Agricultural University, Indonesia, » World Patent Information, vol. 36, p. 22e31, 2014.

[3] C. M. Mejía, La Gestión Universitaria de la Propiedad Intelectual en Colombia, Bogotá: Bonaventuriana, 2015.

[4] G. A. Garcia, «La propiedad intelectual en las economías universitarias, " Revista FACULTAD DE DERECHO Y CIENCIAS POLÍTICAS, vol. 38, no 108, pp. $53-72,2008$.

[5] D. González y A. Jaime, «El Patentamiento Universitario en Colombia," Journal of Technology Management \& Innovation, vol. 8 , no Special Issue ALTEC, pp. 233-245, 2013.

[6] N. Cheeptham y P. Chantawannakul, «Intellectual property management and awareness at the university level in the biotechnology era: a Thai perspective," World Patent Information, vol. 23, pp. 373-389, 2001.
[7] Universidad Industrial de Santander, «PILA Network: La Red de Propiedad Intelectual e Industrial en Latinoamérica recuento de 3 años de colaboración.,» 2011. [En línea]. Available: http:// pila-network.org/sites/default/files/Version_Pdf_ del_Libro.pdf. [Último acceso: 14 Enero 2017].

[8] D. González, «La Gestión de la Propiedad Intelectual en las Universidades Colombianas: El caso de la Universidad Industrial de Santander," Tesis de Grado - Universidad Industrial de Santander, Bucaramanga, 2012.

[9] R. Welsh, L. Glenna, W. Lacy y D. Biscotti, «Close enough but not too far: Assessing the effects of university-industry research relationships and the rise of academic capitalism, " Research Policy, no 37, p. 1854-1864, 2008.

[10] K. Luna y J. Solleiro, "La gestión de la propiedad intelectual en Centros de Investigación Mexicanos: El caso del Instituto Mexicano del Petróleo, » Journal of Technology Manag e Innovation, vol. 2, no 2, pp. 157- 169, 2007.

[11] Red PILA, «http://pila-network.org/,» 2017. [En línea]. Available: http://pila-network.org/presentaci\%C3\%B3n. [Último acceso: 16 febrero 2017].

[12] Red PILA, «http://pila-network.org/publicaciones,» 2017b. [En línea]. Available: http://pila-network.org/publicaciones. [Último acceso: 30 Enero 2017].

[13] G. Sabino y M. Ayuso, «La propiedad intelectual en las universidades brasileñas: Universidad Federal 
de Goias y Universidad de Brasília," Biblios, № 47, pp. 82-103, 2012.

[14] K. Pineda, M. E. Morales y M. C. Ortiz, «Modelos y mecanismos de interacción universidad-empresa-Estado: retos para las universidades colombianas,》 Equidad \& Desarrollo, no 15, pp. 41-67, 2011.

[15] L. Solano, «La Propiedad Intelectual en las Universidades: Nuevos centros de innovación, emprendimiento y transferencia de tecnología; titularidad de derechos y legislación.,» Pontificia Universidad Javeriana, Bogotá, 2012.

[16] Ruta N; Cámara de Comercio de Medellín para Antioquia; Coroporación Tecnnova UEE, Guía Estratégica de Propiedad Intelectual. Universidad Empresa, Medellín : Vallejo Editores, 2014.

[17] L. A. Briceño y E. Morales, «Desafíos de la política pública colombiana frente a la transferencia de resultados de investigación universitaria,» Revista Virtual Via Inveniendi et ludicandi, vol. 10, № 1, pp. 43-86, 2015.

[18] F. Alvarado-Benavides, " Algunos efectos de la gestión tecnológica en la producción industrial y el papel de la educación para su apropiación en Colombia., In Ingeniería Investigación Y Desarrollo, vol. 3, no 2, pp. 23-29, 2006
[19] F. Vallejo y D. Álvarez, «Importancia de la propiedad intelectual en la Universidad Católica de Colombia,» Studiositas., vol. 3, no 1, pp. 21-27, 2008.

[20] Red PILA, «Análisis del nivel de concientización y uso de la PI en las IES: Necesidades Formativas. Colombia,» 2009.

[21] D. González y A. Jaime, «El estado de La Propiedad Intelectual en las Universidades Colombianas, " UIS Ingenierías, vol. 10, no 2, pp. 101-112, 2011.

[22] M. E. Morales, R. Sanabria y P. A. Plata, «Determinantes de la transferencia de propiedad industrial al sector productivo en universidades públicas colombianas," CUADERNOS DE ADMINISTRACIÓN, 2014.

[23] F. Montoya y M. Villalba, «Diagnóstico del Sistema de Gestión de la Propiedad Intelectual Universitaria: Caso Universidad de San Buenaventura," de 5o Congreso Internacional de Gestión Tecnológica y de la Innovación - COGESTEC, Bucaramanga, 2016.

[24] L. Gaviría y J. D. Londoño, «Importancia de un Modelo de Gestión de Propiedad Intelectual en una Institución De Educación Superior: Caso Instituto Tecnológico Metropolitano," de 5o Congreso Internacional de Gestión Tecnológica y de la Innovación - COGESTEC, Bucaramanga, 2016. 